\title{
Design of Composable Proxy Filters for Heterogeneous Mobile Computing
}

\author{
Philip K. McKinley * and Udiyan I. Padmanabhan \\ Department of Computer Science and Engineering \\ Michigan State University \\ East Lansing, Michigan 48824 \\ \{mckinley,padmana3\}@cse.msu.edu
}

\begin{abstract}
RAPIDware is a collection of software design techniques for constructing adaptive middleware to support collaborative computing. This paper describes an experimental study in the design of adaptive proxy services for resource-limited mobile hosts. The approach is based on detachable Java I/O streams, which enable proxy filters and transcoders to be dynamically inserted, deleted, and reordered on a given data stream. Unlike conventional Java I/O streams, detachable streams can be stopped, disconnected, reconnected, and restarted. As such, they provide the "glue" needed to to support the dynamic composition of proxy services. The operation and implementation of detachable streams are described, followed by a discussion of their use in demanddriven forward error correction services for wireless hosts.
\end{abstract}

Keywords: adaptive middleware, heterogeneous collaborative computing, mobile hosts, wireless local area networks, forward error correction, object-oriented design.

\section{Introduction}

The large-scale deployment of wireless communication services and advances in portable computers are quickly making "anytime, anywhere" computing a reality. One class of applications that can benefit from this expanding and varied infrastructure is collaborative computing. Examples include computer-supported cooperative work, computer-based instruction, collaborative scientific experimentation, mobile operator support in industrial installations, and crisis management systems. A diverse infrastructure enables individuals to collaborate via widely disparate technologies, some using workstations on high-speed local area networks (LANs), and others using wireless handheld/wearable devices.

* This work was supported in part by the NSF grants CDA-9617310, NCR-9706285, CCR-9912407, and EIA-0000433.
Collaborative applications differ widely in their qualityof-service requirements and, given their synchronous and interactive nature, they are particularly sensitive to the heterogeneous characteristics of both the computing devices and the network connections used by participants. One approach to accommodating heterogeneity is to introduce middleware layer between applications and underlying transport services. The appropriate middleware framework can not only help to hide differences among networks and devices, but can facilitate the development of new applications through software reuse and extensibility.

We previously developed Pavilion [15], an objectoriented middleware framework for collaborative webbased applications. Pavilion enables a developer to construct new applications by inheriting and extending its default functionality. Like other collaborative computing frameworks for mobile users, Pavilion uses proxy servers to accommodate the limitations of mobile hosts and the variable conditions of wireless links.

In a follow-on project, we are developing RAPIDware, a collection of software design methods and supporting classes for adaptive middleware. A major goal of RAPIDware is to enable middleware frameworks to dynamically instantiate new components in response to external events. The RAPIDware methodology applies not only to communication protocols, but also to fault tolerance components, security services, and reconfigurable user interfaces.

For RAPIDware to achieve these goals, it must be possible to compose middleware functionality "on the fly." In the case of proxies that support mobile hosts, for instance, a framework is needed that enables filters and transcoders to be dynamically inserted, deleted, and reordered on a running data stream. Moreover, the framework will be most flexible if these components need not be compiled into the proxy code a priori, but rather can themselves be mobile components that are uploaded into proxies at run time.

This paper describes our initial work in this area, namely, the development and use of detachable Java I/O streams. Unlike conventional Java I/O stream classes, detachable 
streams can be stopped, disconnected, reconnected, and restarted. As such, they provide the "glue" needed to to support the dynamic composition of proxy services. In the remainder of the paper, we review the Pavilion and RAPIDware projects, describe the basic operation and implementation of detachable streams, and discuss how we are using them to realize adaptive proxy services for mobile hosts. Due to space limitations, many details of the project are omitted here, but can be found in a companion technical report [17].

\section{Background}

In this section, we briefly review the Pavilion project, describe the goals of the RAPIDware project, and discuss how composable proxy filters can help to attain those goals.

Pavilion. In order to explore the design and operation of middleware for heterogeneous collaborative computing, we previously developed an object-oriented middleware framework called Pavilion [15], which supports synchronous web-based collaboration. As illustrated in Figure 1, Pavilion can be used in a default mode, in which it operates as a collaborative web browser. A browser interface component monitors the activities of the leader's web browser and multicasts URL requests to corresponding interface components on receiving systems; the requested resources themselves are multicast by the leader's HTTP proxy as they are retrieved from the network. In addition to collaborative browsing, Pavilion enables a developer to construct new multi-party applications by reusing and extending existing components: browser interfaces, communication protocols, a leadership protocol for session floor control, and a variety of proxy servers. The Pavilion framework itself is written in Java, but supports components written in other languages, including off-the-shelf software such as Netscape Navigator, Internet Explorer, and virtually any helper application for displaying a particular media type. Details can be found in $[12,15]$.

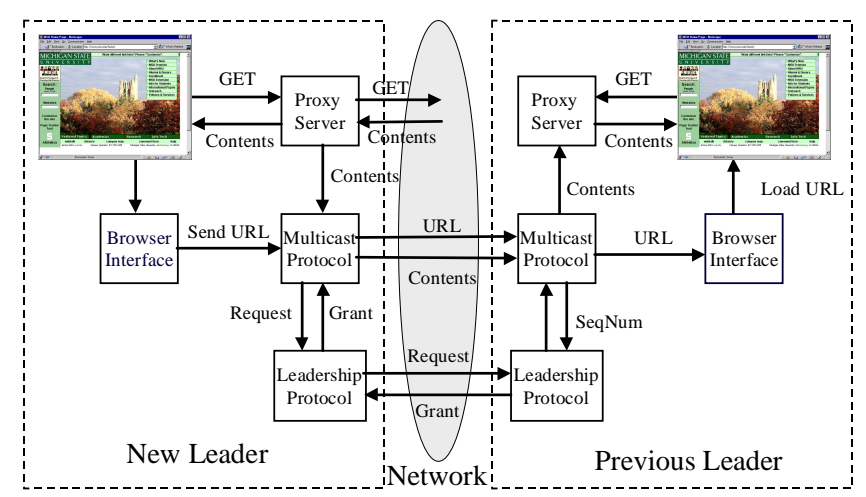

Figure 1. Pavilion-based collaborative browsing.
An important issue in collaborative computing, particularly for heterogeneous environments, is the offloading of functionality from the participating hosts onto proxy servers [1,3-5, 21, 25]. In Pavilion, proxy duties include transcoding and filtering of data streams to reduce bandwidth and load on mobile clients [2], data caching for memory-limited handheld devices [14], and forward error correction for realtime isochronous communication $[7,13]$ and reliable data delivery [16] on wireless networks.

RAPIDware. The RAPIDware project extends Pavilion by automating the instantiation and reconfiguration of middleware components, such as proxies, in order to accommodate resource-limited hosts and dynamic network conditions. A key principle in RAPIDware is to separate adaptive middleware components from non-adaptive, or core, middleware services, thereby facilitating dynamic reconfiguration of components at run time. Towards this end, we are designing an extensible set of adaptive components, which we refer to as raplets. Figure 2 depicts a simple example of the intended relationship among raplets and other components in a collaborative application. Shown are three types of systems connected by several data streams. Two of the systems use proxy servers to transcode or otherwise modify data prior to its transmission on wireless links. The middleware layer uses two main types of raplets, observers and responders, to accommodate heterogeneity and adapt to variations in conditions. The observers collectively monitor the state of the system. When an observer detects a relevant event, the observer either instantiates a new responder or requests an extant responder to take appropriate action to address the event. Example events include changes in the quality of a network connection, disparities among collaborating devices, and changes in user/application preferences or policies. Responder raplets are programmed to handle such events by instantiating new components or modifying the behavior of a communication protocol or user interface.

Given the variety of mobile devices and networks, as well as differences in user preferences, it is necessary that proxy services be composed and modified dynamically. For example, a proxy that is created when a user joins a particular collaborative session may later need to adapt to a changing environment. External factors include dynamic conditions on a given wireless link, changes in network characteristics due to user migration across network cells, and changes in capabilities as the application is handed off from one computing device to another.

Recently, several middleware projects have addressed the issue of configurable and adaptive proxy services for mobile hosts $[1,19,21]$. These designs enable the flow of data to be filters and transcoders to be configured at run time in order to match the capabilities of users devices and networks (see Section 6). If all possible data filters are known to the proxy a priori, then this task is relatively simple. 


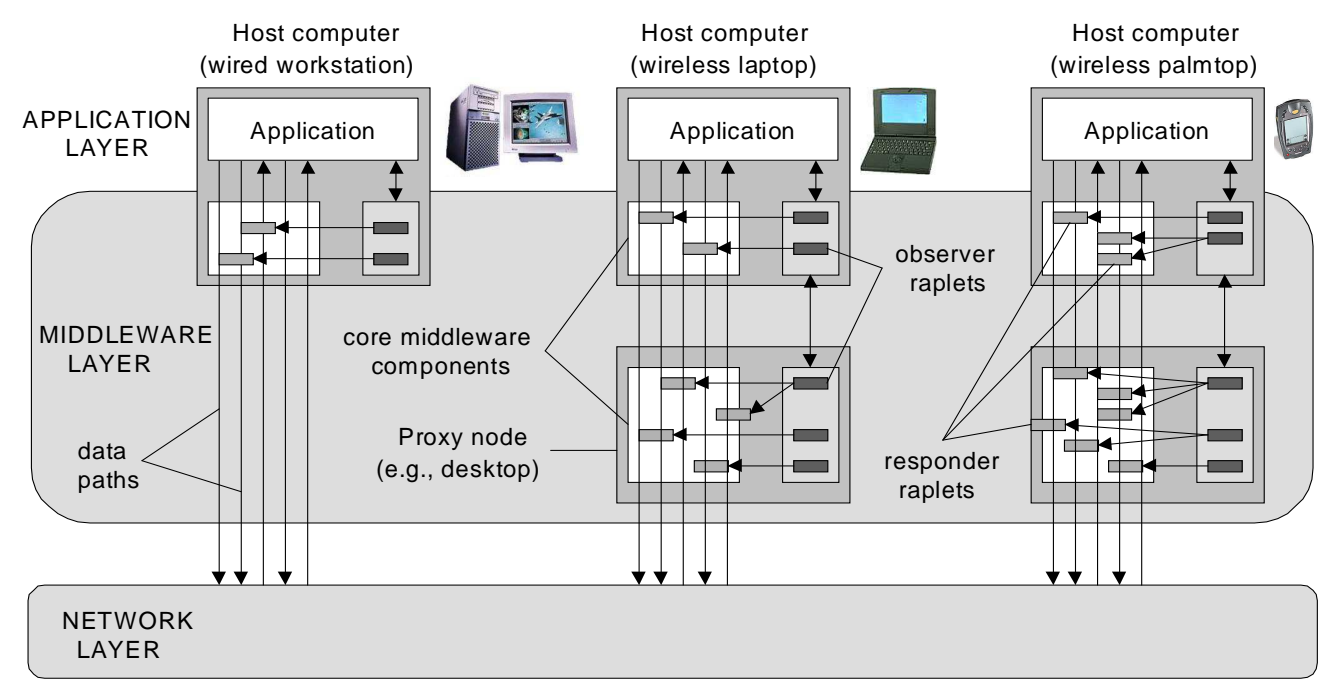

Figure 2. Configuration of RAPIDware adaptive middleware components.

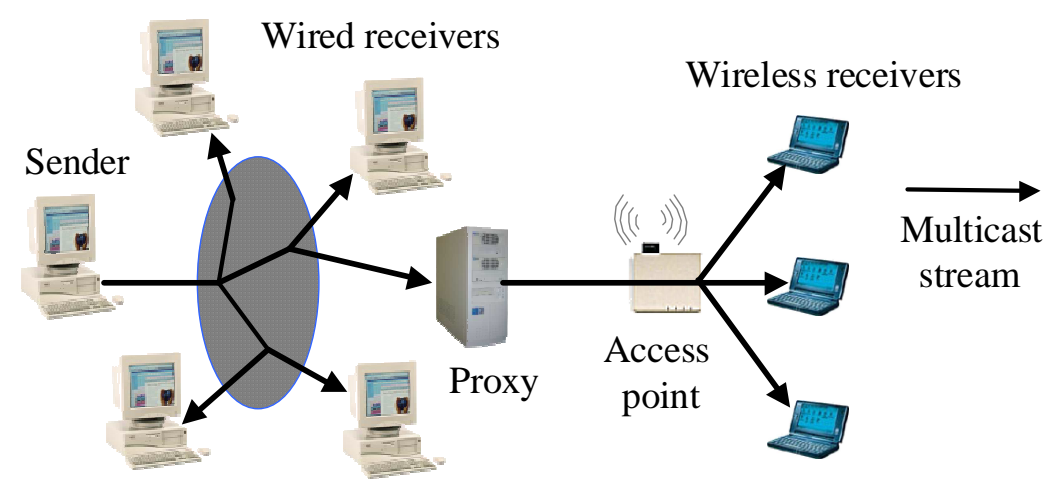

Wired receivers

Figure 3. Proxy configuration for nodes on a wireless LAN.

However, this might not always be the case. Hence, we seek to create a framework that enables RAPIDware-compatible filters to be developed by third parties, and dynamically inserted into an existing proxy by application processes. To do so, we require a set of interfaces and objects that enable a stream of data to be disconnected and redirected to another piece of code, without relying on participation from the ultimate endpoints of the stream, and without compromising the integrity of the data. In the remainder of this paper, we describe a method of providing this functionality that is based on a new type of Java I/O stream.

\section{Proxy Configuration}

To illustrate the operation of RAPIDware proxy components, let us consider an example. Figure 3 depicts the physical configuration of a RAPIDware proxy that we used in an experimental study. The proxy node supports mobile users, connected via a wireless LAN, who are participating in a collaborative session with other users on the wired network. Suppose that this proxy receives a live video stream on a socket, transcodes the stream to a lower bandwidth format, and forwards the resulting data to one or more wireless handheld computers. Now let us assume that the user wants to maintain the connection as she moves from her office (near the access point) to a conference room down the hall. Our experiments show that packet loss rate can change dramatically over a distance of several meters on wireless LANs [16].

When losses rise above a given level, the RAPIDware system should insert an FEC filter into the video stream. However, the insertion should not disturb the connection to 
the source of the stream. Moreover, since the FEC filter may be specific to video streams (e.g., placing more redundancy in I frames than in B frames [24]), we need to consider the format of the stream in order to start the FEC filter at a "frame boundary" in the stream. Finally, it is possible that the application itself was dynamically downloaded to the mobile host, in which case the associated filter for low-bandwidth connections may not have been known to the proxy in advance.

This example illustrates several key requirements of our framework: insertion/deletion of dynamically uploaded filters on a pre-existing streams, insertion at points specific to the stream type, and transparent operation with respect to connections involving non-RAPIDware participants.

To meet these requirements, our first task was to design supporting objects and interfaces to enable filters to be inserted, deleted, and chained together. Figure 4 depicts the resulting software structure of a RAPIDware proxy and its operation on a single data stream. The proxy receives and transmits the stream on EndPoint objects, which encapsulate the actual network connections. Each EndPoint has an associated thread which reads or writes data on the network, depending on the configuration of the EndPoint. The network sockets that are encapsulated within EndPoints can persist as long as they are needed.

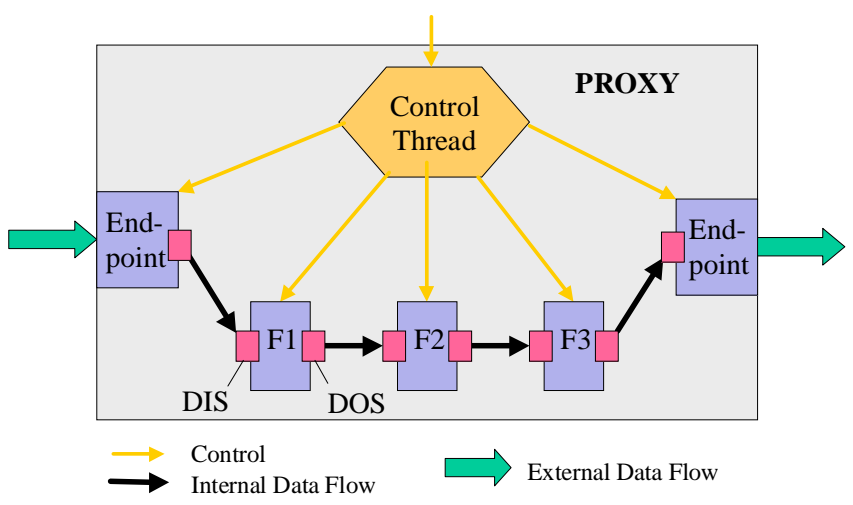

Figure 4. Composition of proxy filters.

A ControlThread object is responsible for managing the insertion, deletion, and ordering of the filters associated with the stream. In this example, the proxy comprises three filters, F1, F2, and F3. The key support mechanisms are detachable stream objects, namely, DetachableInputStream (DIS) and DetachableOutputStream (DOS). The DIS and DOS are used for all communication among filters, and between filters and EndPoints. Unlike their piped counterparts, however, the DIS and DOS can be stopped (paused), disconnected, and reconnected, enabling the dynamic redirection and modification of data streams.

\section{Class Definitions and Implementations}

In this section, we describe each of the major classes used to implement composable proxy filters.

DetachableOutputStream and DetachableInputStream. These classes are based on the the Java PipedOutputStream and PipedInputStream classes, respectively. DetachableOutputStream extends the base java.io.OutputStream class, and DetachableInputStream extends the base java.io.InputStream class. In addition to overriding most of the base class methods, we have also included additional state variables and methods to implement the functionality needed to support composable filters, namely, the ability to pause and reconnect the flow of data to another data source or sink.

Figure 5 illustrates the relationship between a DetachableOutputStream (DOS) and a DetachableInputStream (DIS). The connect() method is used to associate a specific output stream with a specific input stream. Among other initializations, the connect() method sets DOS.sink and DIS.source variables so as to identify the other half of the connection. The DIS.connect() method simply calls the DOS.connect method, which actually does this initialization.

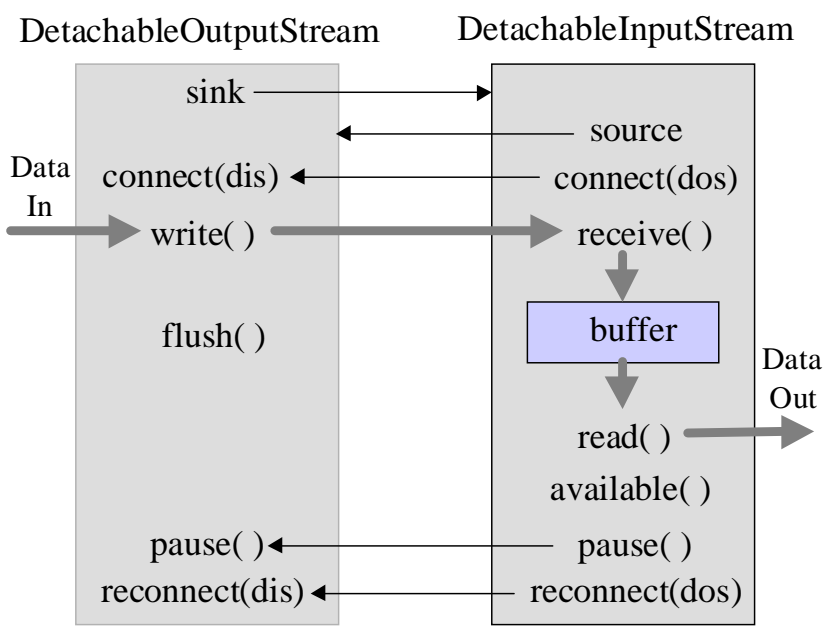

Figure 5. Configuration of detachable streams.

As with their piped counterparts, the data written to the stream is buffered at the DIS side. An invocation of the DOS.write() method results in a call to the DIS.receive() method, which places the data in the buffer. The DIS.available() method returns the number of bytes currently in the buffer, and data is retrieved from the buffer using the DIS.read() method. The DOS.flush() method can be used to force any buffered output bytes to be written out, and notifies any readers that bytes are waiting in the pipe.

Unlike the PipedOutputStream and PipedInputStream 
classes, both the DOS and DIS can be temporarily paused and reconnected to other streams. The pause() method has to be called before any actual disconnection and switching of the data stream. The pause() method blocks writes to the buffer and ensures that all the data has been read from the buffer. It also sets flags indicating that the two sides are no longer connected. A global variable swflag is used to indicate that the stream is being paused and "switched." As with connect(), the DIS.pause() method in the DIS is actually a reference call to the DOS.pause(). Code excerpted from DOS.pause() follows:

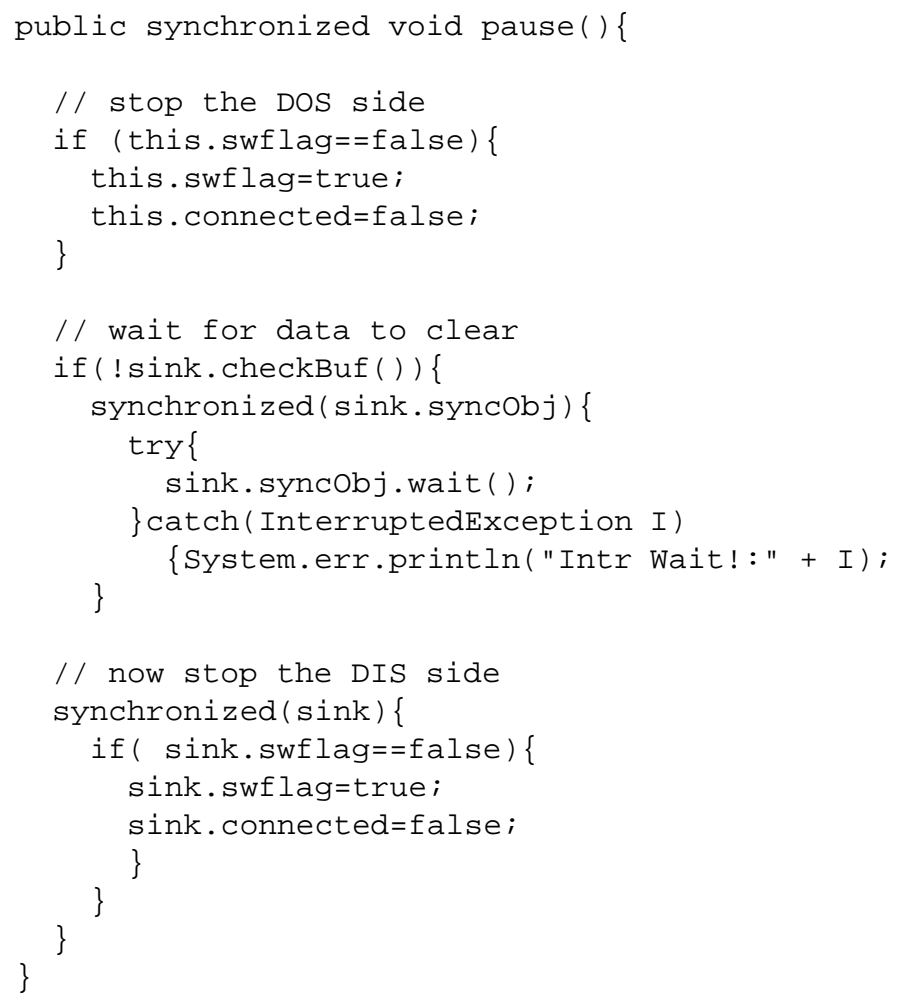

Once the pause method returns, the reconnect() method can be used to attach the DIS and DOS to other stream components. Again, the DIS.reconnect() is a reference call to the DOS.reconnect(). If the buffer has not yet emptied, the caller is suspended to wait on the syncObject of the DIS, which is released when the buffer becomes empty. The reconnect() method checks whether the call is valid (not still in the connected state) and then mimics the actions of the connect() method in setting several global variables, as shown below:

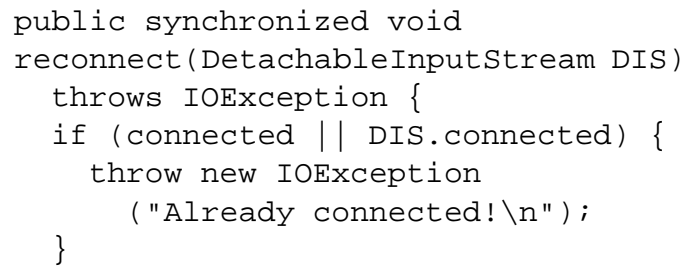

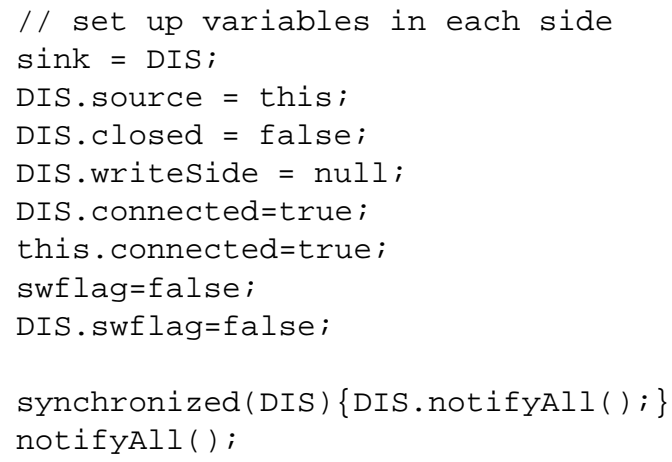

Filter and FilterContainer. The Filter class is a base class that is meant to be extended by all proxy filters that are to be run in the proposed framework. The class contains an instance of DIS and DOS that are always present. The ControlThread uses the DIS and DOS to manipulate the stream connections. A group of methods (e.g., setDIS, setDOS, getid) is used to establish references to the the DIS and DOS in the filter code itself. The FilterContainer class is used to hold an array of Filter objects. This functionality is required when we upload new objects into the framework. The FilterContainer class has methods to obtain the number of Filters available and an enumeration method to return a String enumeration of the Filter objects names.

ControlThread. This class is used to manage the configuration of filters on a given stream supported by the proxy. The class maintains the Filter Vector, a dynamic array that holds references to the currently configured filters. The class implements methods to insert and delete filters from the Filter Vector, as well as methods that allow the ControlManger class to query about the available Filters and the methods they support. The ControlThread receives commands from across the network, either from the mobile client, from a application server, or from the control manager. The following code segment is excerpted from the add() method, which inserts a filter at an indexed location in a running stream.

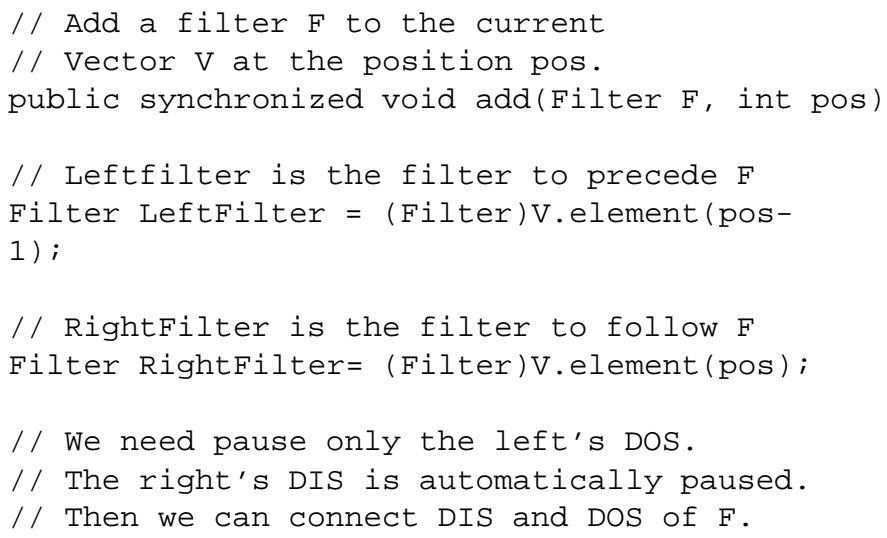




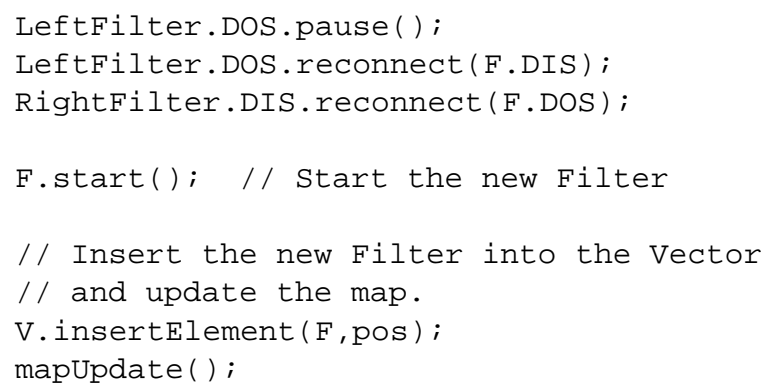

EndPoint. As discussed earlier, EndPoints are special extensions of Filters that are instantiated by the ControlThread for providing Input and Output services to the framework. If the I/O is network-based, then the EndPoint objects would be a EndPointSocketReader and EndPointSocketWriter. If the $\mathrm{I} / \mathrm{O}$ is a non-network stream then we would use an EndPointStreamReader and EndPointStreamWriter. Each EndPoint contains an active thread that handles I/O to/from the proxy. Combined with the ControlThread, two EndPoints comprise a "null" proxy, that is, one that simply forwards data without modifying it. Upon insertion of a filter between the EndPoints, the stream is redirected through the new code.

ControlManager. As described earlier, it is our goal that much of the adaptive functionality of RAPIDwarebased systems will execute automatically, based on predefined user preferences and device/network descriptors. To test the behavior of RAPIDware components, however, we found it useful to develop a user interface that can be used to manage RAPIDware-based collaborative sessions. The ControlManager class is a Swing-based GUI designed for this purpose. The ControlManager supports management of multiple proxies. Based on responses to queries, it constructs a graphical representation of the state of the proxy, including the current configuration of filters, based on the methods available in the ControlThread. This design enables the same ControlManager to be used with different types of proxies. The GUI has pull down menus and dialog text boxes that allow an administrator to insert and remove filters at specified locations in a given stream. The ControlManager uses serialization of filter objects to deliver new filters to the proxy, as requested.

\section{Example: Forward Error Correction}

In the earlier Pavilion project, we constructed proxies to perform several functions on behalf of mobile host: caching, transcoding, and insertion of FEC information. We are in the process of porting these proxy components to the RAPIDware framework so that they can be instantiated and reconfigured at run time in response to external events.

The first proxy we have integrated into the RAPIDware framework is one that introduces FEC to live audio streams prior to transmission on wireless LANs [13]. The filter uses $(n, k)$ block erasure codes [20], which convert $k$ source packets into $n$ encoded packets, such that any $k$ of the $n$ encoded packets can be used to reconstruct the $k$ source packets. The advantage of using block erasure codes for multicasting is that a single parity packet can be used to correct independent single-packet losses among different receivers.

Figure 6 shows the components that constitute the FEC audio proxy. Each component comprises one or more threads in the implementation. Let us first consider delivery of an audio stream to mobile users. The WiredReceiver object receives multicast data packets over the wired network and delivers them to a PacketBuffer. The FEC_Encoder collects the data packets into FEC data blocks of size $k$. When a group of $k$ packets is full, encoding routines are invoked to produce $n-k$ parity packets. Both the data and parity packets are forwarded to the Wireless Sender object, which uses IP multicast to transmit them on the WLAN. To enable mobile users to speak to other group members, the proxy also contains a WirelessReceiver object, which receives data and parity packets on the wireless network interface. An FEC_Decoder object extracts the audio data and forwards the resulting packets to a WiredSender object, which forwards them to participants accessible via the wired network.

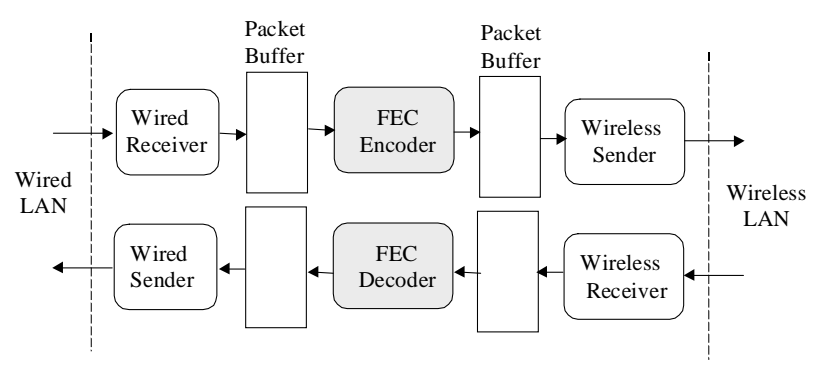

Figure 6. Operation of FEC audio proxy.

Porting this filter to the RAPIDware framework was straightforward by replacing the socket interfaces of the original proxy with detachable streams. Figure 7 shows a sample of the results of the experiments on our testbed. We recorded audio in Windows PCM-based waveform audio file format (.WAV) at a rate of 8000 samples per second for two 8-bit/sample stereo channels and transmitted it through a proxy to three wireless laptop computers. These tests used our $2 \mathrm{Mbps}$ WaveLAN network. In Figure 7, we plot raw and reconstructed receipt rate at a location 25 meters from our access point, using a $(6,4)$ FEC configuration (we use small groups so as to minimize jitter). The average raw receipt rate is already quite high, $98.54 \%$, but even these small losses result in noticeable degradation in the quality of the audio. Using FEC, the reconstructed packet rate increases to nearly $100 \%$, producing very clear audio quality. Addi- 
tional details on performance can be found in [13].

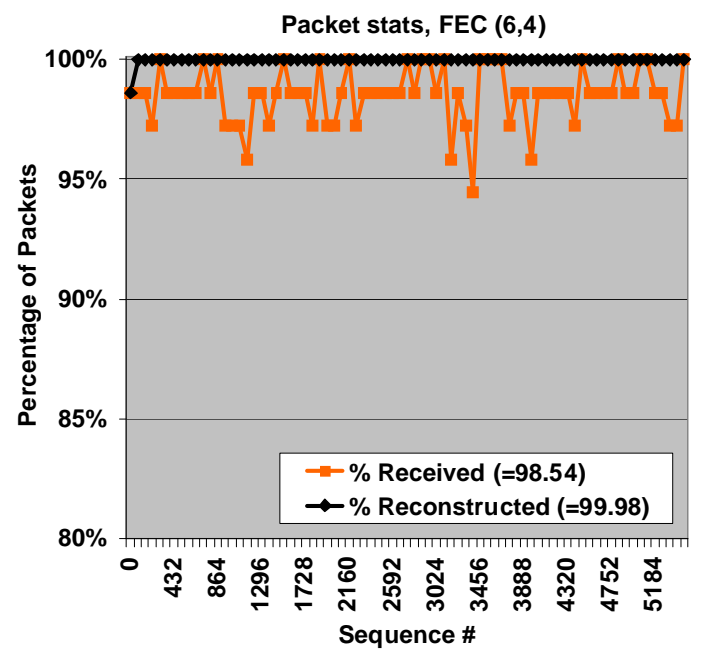

Figure 7. Trace data for $\operatorname{FEC}(6,4)$ audio $\mathrm{FEC.}$

\section{Related Work}

Recently, several research groups have started to address the issue of adaptive middleware frameworks that can accommodate dynamic, heterogeneous infrastructures. Examples include Rover [8], BARWAN [19], MASH [11], TAO [9], Mobiware [1], the Middleware Control Framework [10], QuO [23], Odyssey [18], and DaCapo++ [22]. These projects have greatly improved the understanding of how middleware can accommodate device heterogeneity and dynamic network conditions, particularly in the area of adaptive communication protocols and services. Indeed, several of these projects address the issue of dynamically configuring proxy functionality. In the remainder of this section, we discuss three such projects and their relationship to RAPIDware.

In the MobiWare project [1], "mobile filters" can be dispatched to various nodes in the network, or to hosts, in order to achieve bandwidth conservation. Apparently, these filters are established only during handoff from one network to another. The detachable streams discussed herein could be used to extend this functionality so that filters could be reorganized at any time.

The Berkeley TranSend proxy is based on the TACC model [6], in which workers are the active components of the proxy. The TACC server enables workers to be chained together in a manner similar to Unix pipes. Details of the implementation are not available. However, the project focuses on proxies built atop highly available parallel workstation clusters, whereas RAPIDware proxies are intended to be lightweight, on-demand proxies established dynamically on one or more idle workstations available to the user.
The Stanford Mobile People Architecture (MPA) [21] is designed to support person-to-person reachability through the use of personal proxies. A key component of the personal proxy is the use of conversion drivers, which are configured dynamically to match the capabilities of the user's device and network. The RAPIDware project seeks to develop a general framework for the design of such software. In this case, the detachable stream mechanism and filter container class may provide a useful mechanism for composing such drivers and facilitating their loading from across the network.

Finally, we emphasize that this paper has described only a small part of the RAPIDware project. A given external event, such as a sudden decrease in quality on a wireless link, can affect not only communication protocols, but also middleware components associated with fault tolerance, security, and user interfaces. The overall goal of the RAPIDware project is to develop an integrated methodology for middleware adaptability that encompasses not only communication services, but fault tolerance actions and user interface reconfiguration. We will report developments in these areas in future papers.

\section{Conclusions and Future Work}

In this paper, we have described our initial studies in the design and use of composable proxy filters to support mobile clients. We described the implementation of detachable Java I/O streams, which enable proxy filters and transcoders to be dynamically inserted, deleted, and reordered at a proxy for mobile hosts. Finally, we discussed the use of this framework to support dynamic instantiation of a forward error correction filter for live audio streams transmitted over wireless LANs.

Our continuing work in this area addresses several issues: porting of additional proxies to the RAPIDware framework; development of language support to characterize the "composability" of filters; and application of RAPIDware concepts to fault tolerance and adaptive user interfaces. Given the increasing presence of wireless networks in homes and businesses, we envision immediate application of the proposed techniques to improve performance of collaborative applications involving users who roam within a wireless environment.

Further Information. A number of related papers and technical reports on the Pavilion and RAPIDware projects can be found at http: / / www. cse.msu.edu/ mckinley.

Acknowledgements. The authors would like to thank Arun Mani, Suraj Gaurav, Peng Ge, and Chiping Tang for their contributions to this work. This project was supported 
in part by the NSF grants CDA-9617310, NCR-9706285, CCR-9912407, and EIA-0000433.

\section{References}

[1] O. Angin, A. T. Campbell, M. E. Kounavis, and R.R.-F.M. Liao. The Mobiware toolkit: Programmable support for adaptive mobile networking. IEEE Personal Communications Magazine, Special Issue on Adapting to Network and Client Variability, August 1998

[2] J. Arango and P. K. McKinley. VGuide: Design and performance evaluation of a synchronous collaborative virtual reality application. In Proceedings of the IEEE International Conference on Multimedia and Expo, July 2000.

[3] B. R. Badrinath, A. Bakre, R. Marantz, and T. Imielinski. Handling mobile hosts: A case for indirect interaction. In Proc. Fourth Workshop on Workstation Operating Systems, Rosario, Washington, October 1993. IEEE.

[4] Y. Chawathe, S. Fink, S. McCanne, and E. Brewer. A proxy architecture for reliable multicast in heterogeneous environments. In Proceedings of ACM Multimedia '98, Bristol, UK, September 1998

[5] L. Chen and T. Suda. Designing mobile computing systems using distributed objects. IEEE Communications Magazine, 35(2), Feb. 1997.

[6] A. Fox, S. D. Gribble, Y. Chawathe, and E. A. Brewer. Adapting to network and client variation using active proxies: Lessons and perspectives. IEEE Personal Communications, August 1998.

[7] P. Ge and P. K. McKinley. Experimental evaluation of error control for video multicast over wireless LANs. In Proceedings of the Third International Workshop on Multimedia Network Systems, Phoenix, Arizona, April 2001.

[8] A. D. Joseph, J. A. Tauber, and M. F. Kaashoek. Mobile computing with the Rover toolkit. IEEE Transactions on Computers: Special issue on Mobile Computing, 46(3), March 1997.

[9] F. Kuhns, C. O'Ryan, D. C. Schmidt, O. Othman, and J. Parsons. The design and performance of a pluggable protocols framework for object request broker middleware. In Proceedings of the IFIP Sixth International Workshop on Protocols For High-Speed Networks (PfHSN '99), Salem, Massachusetts, August 1998

[10] B. Li and K. Nahrstedt. A control-based middleware framework for quality of service adaptations. IEEE Journal of Selected Areas in Communications, 17(9), September 1999.

[11] S. McCanne, E. Brewer, R. Katz, L. Rowe, E. Amir, Y. Chawathe, A. Coopersmith, K. Mayer-Patel, S. Raman, A. Schuett, D. Simpson, A. Swan, T. Tung, D. Wu, and B. Smith. Toward a common infrastructure for multimedianetworking middleware. In Proc. 7th Intl. Workshop on Network and Operating Systems Support for Digital Audio and Video (NOSSDAV '97), St. Louis, Missouri, May 1997.

[12] P. K. McKinley, R. R. Barrios, and A. M. Malenfant. Design and performance evaluation of a Java-based multicast browser tool. In Proceedings of the 19th International Conference on Distributed Computing Systems, pages 314-322, Austin, Texas, 1999.
[13] P. K. McKinley and S. Gaurav. Experimental evaluation of forward error correction on multicast audio streams in wireless LANs. In Proceedings of ACM Multimedia 2000, pages 416-418, Los Angeles, California, November 2000.

[14] P. K. McKinley and J. Li. Pocket Pavilion: Synchronous collaborative browsing for wireless handheld computers. In Proceedings of the IEEE International Conference on Multimedia and Expo, July 2000.

[15] P. K. McKinley, A. M. Malenfant, and J. M. Arango. Pavilion: A distributed middleware framework for collaborative web-based applications. In Proceedings of the ACM SIGGROUP Conference on Supporting Group Work, pages 179_ 188, November 1999.

[16] P. K. McKinley and A. P. Mani. An experimental study of adaptive forward error correction for wireless collaborative computing. In Proceedings of the IEEE 2001 Symposium on Applications and the Internet (SAINT-01), San DiegoMission Valley, California, January 2001.

[17] P. K. McKinley and U. I. Padmanabhan. Design of composable proxy filters for wireless collaborative computing. Technical Report MSU-CSE-00-26, Computer Science and Engineering, Michigan State University, East Lansing, Michigan, November 2000

[18] B. D. Noble and M. Satyanarayanan. Experience with adaptive mobile applications in Odyssey. Mobile Networks and Applications, 4:245-254, 1999.

[19] R. H. Katz, E. A. Brewer, et al. The Bay Area Research Wireless Access Network (BARWAN). In Proceedings Spring COMPCON Conference, 1996.

[20] L. Rizzo. Effective erasure codes for reliable computer communication protocols. ACM Computer Communication Review, April 1997.

[21] M. Roussopoulos, P. Maniatis, E. Swierk, K. Lai, G. Appenzeller, and M. Baker. Person-level routing in the mobile people architecture. In Proceedings of the 1999 USENIX Symposium on Internet Technologies and Systems, Boulder, Colorado, October 1999.

[22] B. Stiller, C. Class, M. Waldvogel, G. Caronni, and D. Bauer. A fexible middleware for multimedia communication: Design implementation, and experience. IEEE Journal of Selected Areas in Communications, 17(9):1580-1598, September 1999.

[23] R. Vanegas, J. A. Zinky, J. P. Loyall, D. A. Karr, R. E. Schantz, and D. E. Bakken. QuO's runtime support for quality of service in distributed objects. In Proceedings of the IFIP International Conference on Distributed Systems Platforms and Open Distributed Processing (Middleware'98), September 1998.

[24] D. Xu, B. Li, and K. Nahrstedt. Qos-directed error control of video multicast in wireless networks. In Proceedings of IEEE International Conference on Computer Communications and Networks, October 1999.

[25] B. Zenel and D. Duchamp. Intelligent communication fi ltering for limited bandwidth environments. In Proc. Fifth Workshop on Hot Topics in Operating Systems, Rosario, Washington, May 1995. 